\title{
Gesellschaft der Augenärzte in Moskau
}

\section{Sitzung vom 27. Oktober (9. November 1909)}

Logetschnikow widmet ehrende Worte dem Andenken des verstorbenen Priv.-Doz. D. A.

Natanson sen.

Hierauf gibt Aiuerbach einen Ueberblick über den Lebenslauf und die wissenschaftliche Tätigkeit desselben.

Fr. Dr. Molodenkowa demonstriert eine Kranke mit beiderseitiger $\mathrm{p} \beta \Gamma$ ipherischer Hornhautektasie. Die ektatischen Partien sind leicht getrübt. von Gefässen durchzogen. Von der normal gekrümmten Hornhant grenzen sich die Ektasien durch weiss gefärbte Streifen ab, entlang denen zwei Gefässe verlaufen. Vis. O. d. = 1,0, Ast. 1,5 D. Vis. O. S. = Finger-zählen 4,5 Met.; Ast. irreg. 18,0 D. Aetiologische Momente konnten nicht ermittelt werden.

Blagowestschensky zeigt eine 60 jährige Kranke mit subkonjunktival luxierter Linse. Vor $13 / 4$ Monaten flog gegen ihr linkes Auge ein Holzstück. Blutungen in das Lidgewebe, Hyphäma, unter der Bindehaut eine stark prominierende Geschwulst. Vis = ] /cs; T. -2. Nach 2 Wochen, als die Blutungen resorbiert waren, sah man eine Skleralruptur oben parallel dem Limbus. Die Linse lag unter der Bindehaut. Vortragender bespricht den Mechanismus der subkonjunktivalen Linsenluxation und die bekannten Massregeln, die für die operative Entfernung luxierter Linsen gelten.

Rschanitzín demonstriert eine 15 jährige Kranke mit seltener Fundus-veränderung links. Anstelle der Papille sieht man einen weissen, scharf begi-enzten und stark prominierenden (4,0 D.) Bezirk. Im unteren äusseren Quadranten (im umgekehrten Biide) desselben Austríttsstelle der Zentral-gefässe, die stellenweise unterbrochen sind und an der Grenze des Bezirke steil abknicken. Die $\lambda$ '"'enen erweitert. Feine radiäre Streifung des Bezirkes. Parallel dem oberen und inneren Bande desselben, noch in seinem Bereicbe, eine bläuliche, nicht scharf hervortretende Linie. 2 Blutungen in der Netzhaut nach innen von der Papille und ein weiss gelblicher Herd nach unten von derselben. Vis. $=0$. Einige Tage zuvor untersuchte der Vortragende die Kranke und konnte damals keine Veränderungen in der Papille finden. Es bestand nur grosse Netzhautablösung. Vis. = Handbewegung. Sorg-fältige allgemeine Untersuchung ergab keine Abweichungen. Für Lues keine Anhaltspunkte. Hb-(1/8halt $95 \mathrm{pCt}$. Vortragender spricht die Vermutung aus, dass es sich hier um eine Erkrankung (wahrscheinlich Tumor) im sklerochotoidealen Kanal handelt.

Gesellschaft für Augenärzte in Moskau. 50̃1

In der Diskussion teilt Dr. Disler mit, dass er die Kranke vor etwa 2 Wochen gesehen und eine Neuroretinitis ungewöhnlicher Art konstatiert hatte. Eine Netzhautablösung habe er nicht gesehen.

Dr. Lütkewitsch bestätigt das Vorhandensein der Netzhautablösung einige Tage vor der Demonstration.

Smirnow zeigt ein 4 jähriges Mädchen mit angeborenen beiderseitigen Kolobomen der Maculae. Das Augenspiegelbild ist typisch für die Kolo-bome dieser Gegend. Das Interesse des Falles liegt 
im beiderseitigen Auf treten der Veränderung. Die Mutter des Mädchens infizierte sich mit Lues im 8. Monate der Schwangerschaft.

A. Natanson jun. zeigt einen Kranken mit Atrophia n. optici nach Orbitalverletzung mit einem eisernen Stab. Gleich nach der Verletzung fand der Vortragende eine kleine, frei für die Sonde durchgängige Bindehaut-wunde, Ptosis. vollständige Unbeweglichkeit des Augapfels, Mydriasis und Reaktionslosigkeit der Pupille. Commotio retinae. Vis. $=0$. Exophthalmus fehlte. Nach 3 Wochen verschwanden alle Erscheinungen der Lähmung. Atrophia n. optici. Vis. $=0$. Es kann zwei Erklärungen für die Entwicklung der Atrophia geben: entweder direkte Verletzung des Nervenstammes durch den eingedrungenen Fremdkörper, oder indirekte, durch Knochen-splitter im Canalis nervi optici. Der eiserne Stab gelangte wahrscheinlich bei der Verletzung bis zu dieser Stelle, sonst wären die Symptome des Mitcrgriffenseins der airderen Nerven nicht zu erklären.

Diskussion.

Strachow weist auf die Gefahren der Sondierung hin. In dem be-schriebenen Falle war ja ein Fremdkörper in der Orbita nicht zu erwarten. Die Sondierung war also hier auch nutzlos. Er teilt ferner 2 Fälle aus eigener Erfahrung mit. Im ersten kam es nach einer Orbitaverletzung zur vollständigen Lähmung der Augenmuskeln. Heilung. Im zweiten bildete sich ein Exophthalmus pulsatilis. Operation beseitigte zwar den letzten, aber es kam zur Atrophia bulbi.

Natanson jun. halt die Sondierung für angezeigt und weist auf einen Fall hin, wo der Fremdkörper nur mittelst Sonde entdeckt wurcle.

Awerbach ist der Meinung, dass es Fälle gibt, wo die Sondierung allein die

Fremdkörperanwesenheit zu bestimmen imstande ist. Der Eingriff ist zwar gefährlich, aber manchmal geradezu unerlässlich. Ferner weist Awerbach auf die Seltenheit der traumatischen Lähmungen der Augenmuskeln hin und führt einen Fall von traumatischer Ptosis (Faustschlagj bei einem Luetischen an. Der andere Fall betrifft eine Frau mit Lähmung des Rectus inferior (Stoss gegen das Auge). In beiden erfolgte glatte Heilung.

Strachow meint, dass die Röntgenoskopie vollkommen die Sondierung ersetzen kann. A $\tau$ verbach: Die Röntgenoskopie kann keine Entscheidung in den Fallen geben, $\lambda$ vo es sich um Holzstücke in der Orbita handelt.

A. Awerbach demonstriert einen 20 jährigen Mann mit subkonjunktî-valer Cyste in der rechten oberen Uebergangsfalte. Keine vorangegangene Konjunktivalerkrankung. Die Probepunktion ergab eine klare. gelbliche Flüssigkeit. Im zentrifugierten Bodensatz neutrophile und polynukleäre Leukozyten und Platten und zylindrisehe Epithelzel,en. DasVorkommen der letzteren spricht dafür dass die Cyste eine lymphatische ist. Dr. F. Odinzow. 